A brai $n$ computer interface based on neur al net work with efficient pre-processing

\begin{tabular}{|l|l|}
\hline 著者 & Nakayana Kenj i , I nagaki Ki yot o \\
\hline $\begin{array}{l}\text { j our nal or } \\
\text { publ i cat i on t i t I e }\end{array}$ & Pr oc. I EEE, I SPACS2006, Yonago, Japan \\
\hline page range & $673-676$ \\
\hline year & 2006-12-01 \\
\hline URL & ht t p: //hdl . handl e. net /2297/6856 \\
\hline
\end{tabular}




\title{
A Brain Computer Interface Based on Neural Network with Efficient Pre-Processing
}

\author{
Kenji Nakayama Kiyoto Inagaki \\ ${ }^{*}$ Graduate School of Natural Science and Technology, Kanazawa Univ. \\ Kakuma-machi, Kanazawa, 920-1192, Japan \\ Tel: +81-76-234-4896, Fax: +81-76-234-4900 \\ E-mail: nakayama@t.kanazawa-u.ac.jp
}

\begin{abstract}
Brain Computer Interface (BCI) is one of hopeful interface technologies between human and machine. However, brain waves are very weak and there exist many kinds of noises. Therefore, what kinds of features are useful, how to extract the useful features, how to suppress noises, and so on are very important.

On the other hand, neural networks are very useful technology for pattern classification. Especially, multilayer neural networks trained through the error back-propagation algorithm have been widely used in a wide variety of field.

In this paper, the neural network is applied to the BCI. Amplitude of the FFT of the brain waves are used for the input data. Several kinds of techniques are introduced in this paper. Segmentation along the time axis for fast response, nonlinear normalization for emphasizing important information with small magnitude, averaging samples of the brain waves for suppressing noise effects and reduction in the number of the samples for achieving a small size network, and so on are newly introduced.

Simulation was carried out by using the brain waves, which are available from the web site of Colorado state university. The number of mental tasks is five. Ten data sets for each mental task are prepared. Among them, 9 data sets are used for training, and the rest one data set is used for testing. Selection of the one data set for testing is changed and accuracy of the correct classifications are averaged over the possible selections. Approximately, $80 \%$ of correct classification of the brain waves is obtained, which is higher than the conventional.
\end{abstract}

\section{INTRODUCTION}

Nowadays, several kinds of interfaces between human and computers or machines have been proposed and developed. For persons being in a healthy condition, the keyboard and the mouse are useful and practical interface. On the other hand, for handicapped persons, several interface techniques, which use available organs and functions, have been studied and developed.

Among the interfaces developed for the handicapped persons, Brain Computer Interface (BCI) has been attractive recently. Brain waves are first analyzed and classified. Mental tasks, which a subject images, are estimated. Furthermore, based on the estimated mental task, computers and machines are controlled [9].

For one application, it can be expected that heavy handicapped persons, who cannot control any parts of their own body, control a wheelchair, computers and other machines through the BCI [6]. Furthermore, in the virtual reality (VR) technology, it may be possible to control a person states in the
VR world, and to have many kinds of experiences in the VR world. For instance, training to avoid danger situations may be possible by using the $\mathrm{BCI}$ technology.

Approaches to the BCI technology includes nonlinear classification by using spectrum power, adaptive auto-regressive model and linear classification, space patterns and linear classification, hidden Markov models, and so on [4]. Furthermore, application of neural networks have been also discussed [3],[5],[7],[10],[11].

In this paper, the multilayer neural network is applied to the BCI. Especially, efficient pre-processing techniques are introduced in order to achieve high probability of correct mental task classification. Simulation were carried out by using the brain waves, which are available from the web site of Colorado state university. Estimation results of the proposed method are compared to the conventional methods [12],[13].

\section{Mental Tasks and Brain Wave Measurement}

\section{A. Mental Tasks}

In this paper, the brain waves, which are available from the web site of Colorado state university [1], are used. The following five kinds of mental tasks are used as imaging.

- Baseline (B)

- Multiplication (M)

- Letter-composing (L)

- Rotation of 3-D object (R)

- Counting numbers (C)

\section{B. Brain Wave Measurement}

Location of the electrodes to measure brain waves is shown in Fig.1. Seven channels including C3, C4, P3, P4, O1, O2, $\mathrm{EOG}$, are used. EOG is used for measuring movement of the eyeballs.

The brain waves are measured for $10 \mathrm{sec}$ and sampled by $250 \mathrm{~Hz}$ for each mental task. Therefore, $10 \mathrm{sec} \times 250 \mathrm{~Hz}=$ 2,500 samples are obtained for one channel and one mental task. One data set includes seven cahnnels. One example of the brain wave is shown in Fig.2.

\section{PRE-PRocessing}

\section{A. Segmentation along Time Axis}

In order to make the BCI responce fast, the brain wave measured during $10 \mathrm{sec}$ is divided into the segments with 


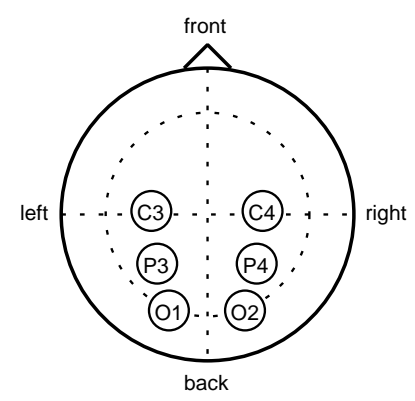

Fig. 1. Location of seven channel electrodes.

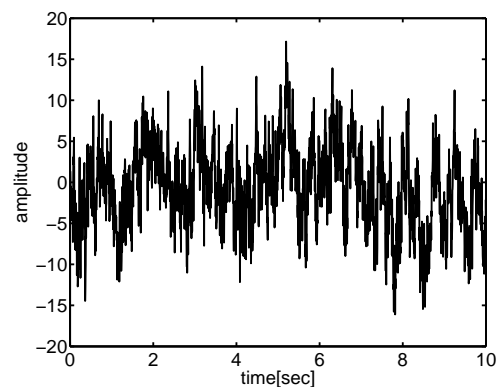

Fig. 2. Example of brain waves.

$0.5 \mathrm{sec}$ length as shown in Fig.3. The segmentation is shifted by $0.25 \mathrm{sec}$. This means the segment with $0.5 \mathrm{sec}$ length can be obtained every $0.25 \mathrm{sec}$.

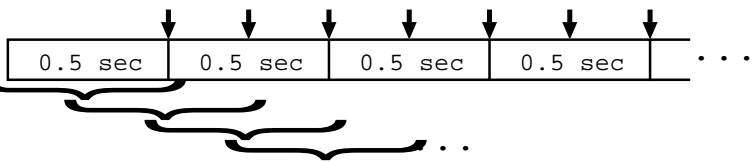

Fig. 3. Segmentation of brain wave along time axis.

\section{B. Amplitude of FFT of Brain Waves}

What kinds of features should be used to classify the brain waves and to estimate the corresponding mental task is very important. In order to avoid effects of time delay, which is not essential, the brain wave is Fourier transformed and its amplitude is used to express feature of the brain wave. The segment of the brain wave with $0.5 \mathrm{sec}$ length includes 125 samples. The amplitude of the FFT of the segment is shown in Fig.4.
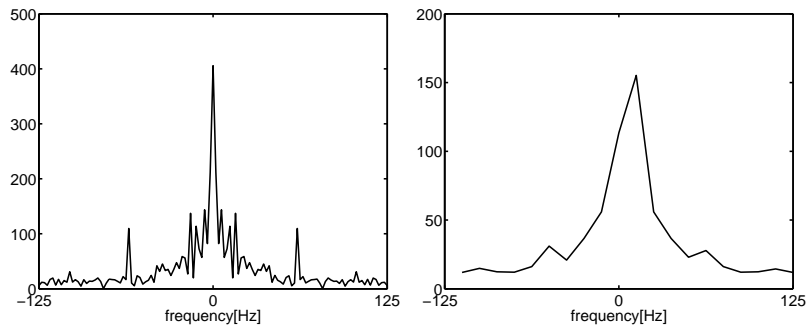

Fig. 4. Amplitude of FFT of segment with $0.5 \mathrm{sec}$ length. Left: 125 samples, Right: 20 samples of averaging.

\section{Reduction of Samples by Averaging}

In order to make the neural network compact and to reduce effects of the noises, the FFT samples in some interval are averaged. The averages are used for the neural network input. The number of samples is reduced to 20 . The amplitude of the FFT for the reduced samples is also shown in Fig.4.

\section{Nonlinear Normalization}

The amplitude of the FFT is widely distributed. Small samples also contain important information for classifying the mental tasks. However, in the neural networks, large inputs play an important role. If large samples do not include important information, correct classification will be difficult. For this reason, the nonlinear normalization as shown in Eq.(1) and Fig.5 is employed in this paper. The small samples are expanded and the large samples are compressed.

$$
f(x)=\frac{\log (x-\min +1)}{\log (\max -\min +1)}
$$

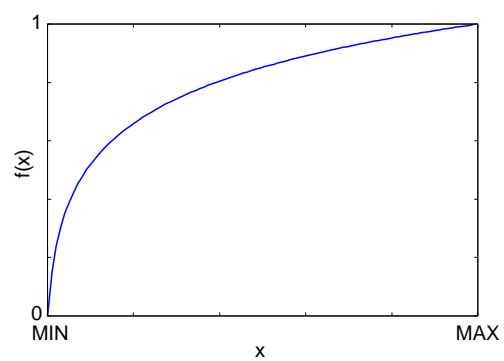

Fig. 5. Nonlinear normalization. Horizontal axis is input and vertical axis is output.

\section{E. Input of Neural Network}

Since the amplitude response is symmetrical, only the right hand side is used. Furthermore, the amplitude response of the seven channels are simultaneously applied to the neural network. An example of the neural network input is shown in Fig.6. In this figure, the left hand side shows the input before the nonlinear normalization, and the right hand side is that after normalization.
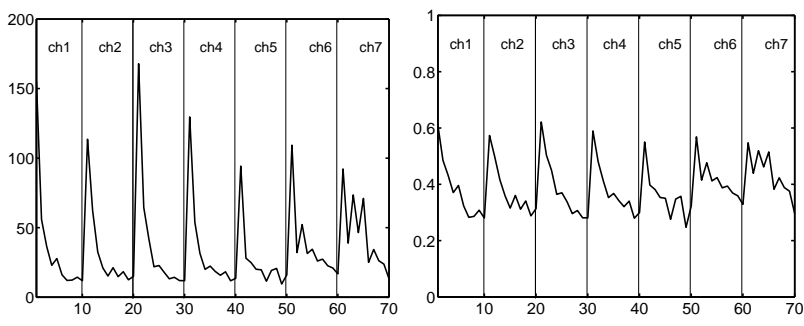

Fig. 6. Input of neural network including 7-channels. Left hand side: Before normalization, Right hand side: After normalization. 


\section{Mental task Classification by Using Neural NETWORK}

A multilayer neural network having a single hidden layer is used. Activation functions used in the hidden layer and the output layer are sigmoid functions. The number of input nodes is 10 samples $\times 7$-channels $=70$. Five output neurons are used for five mental tasks. Therefore, only one output neuron will respond to the applied input data. In the training phase, the target for the output is binary, like $(1,0,0,0,0)$. In the testing phase, the maximum output becomes the winner and the corresponding mental task is assigned. However, when the winner have small value, estimation becomes incorrect. Therefore, the answer of the neural network is rejected, that is any mental task cannot be estimated. The error back-propagation algorithm is employed for adjusting the connection weights.

\section{Simulation CONDitions}

\section{A. Training and Testing Brain Waves}

The brain waves with $10 \mathrm{sec}$ length for five mental tasks were measured 10 times. Therefore, 10 data sets are available. Among them, 9 data sets are used for training and the rest one data set is used for testing. Five data sets are selected as the testing data set. Thus, 5 independent trials were carried out, and classification accuracy is evaluated based on the average over 5 trials [2].

\section{B. Probability of Correct and Error Classifications}

Estimation of the mental tasks is evaluated based on probability of correct classification $\left(P_{c}\right)$ and error classification $\left(P_{e}\right)$.

$$
\begin{aligned}
P_{c} & =\frac{N_{c}}{N_{t}} \\
P_{e} & =\frac{N_{e}}{N_{t}} \\
N_{t} & =N_{c}+N_{e}+N_{r} \\
\text { Rate } & =\frac{P_{c}}{P_{c}+P_{e}}
\end{aligned}
$$

In the above equations, $N_{c}$ is the number of correct classifications, $N_{e}$ is the number of error classifications, and $N_{r}$ is the number of rejections.

\section{Parameters in Neural Network Learning}

- The number of hidden neurons: 20

- A learning rate: 0.2

- Initial weights: Random numbers in $-0.1 \sim+0.1$

- The threshold for rejection: 0.8

- The number of iterations: 5000

\section{Simulation Results}

\section{A. Effects of Number of Samples}

Before the segmentation, effects of the number of samples, that is the amplitude of FFT, is investigated. The successive samples are averaged and this average is used to express the amplitude. The number of samples is reduced from 2,500 to $250,100,50,20$. Since, the amplitude of FFT for real signal is symmetrical, then a half number of them is actually used. Figure 7 shows the learning curves for mental task classification. In this figure, fcorrect classification ratef, that is $P_{c}$, is the average over five mental tasks. From this result, the number of samples of 20 is best.

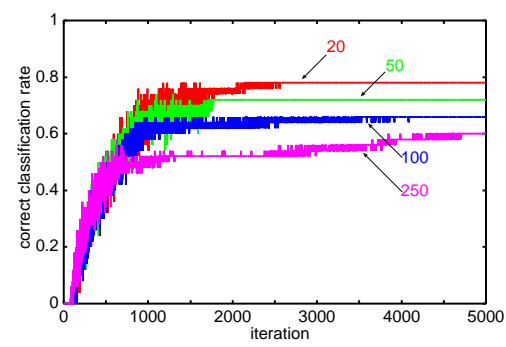

Fig. 7. Learning curves of mental classification for training and testing data. The number of samples is varied from 250 to 20 .

Table I shows the number of correct and error classifications and their probability.

TABLE I

NUMBER OF CORRECT AND ERROR CLASSIFICATIONS AND THEIR PROBABILITY WITH 20 SAMPLES.

\begin{tabular}{|c||c|c|c|c|c|r||c|c|}
\hline Mental Task & $\mathrm{B}$ & $\mathrm{M}$ & $\mathrm{L}$ & $\mathrm{R}$ & $\mathrm{C}$ & Reject & $P_{c}$ & $P_{e}$ \\
\hline Baseline & 7 & 0 & 0 & 0 & 0 & 3 & 70.0 & 0.0 \\
\hline Multiplication & 0 & 8 & 0 & 0 & 0 & 2 & 80.0 & 0.0 \\
\hline Letter-composing & 0 & 0 & 7 & 0 & 0 & 3 & 70.0 & 0.0 \\
\hline Rotation & 0 & 0 & 0 & 8 & 0 & 2 & 80.0 & 0.0 \\
\hline Counting & 0 & 0 & 0 & 0 & 9 & 1 & 90.0 & 0.0 \\
\hline
\end{tabular}

\section{B. Effects of Segmentation}

Aim of the segmentation is to make the $\mathrm{BCI}$ response fast. On the other hand, the length is limited to $0.5 \mathrm{sec}$ for example. In this section, effects of this short length is investigated. As described in the previous section, The $10 \mathrm{sec}$ length of the brain waves is divided into $0.5 \mathrm{sec}$, which has 125 samples. The learning curves are shown in Fig.8.

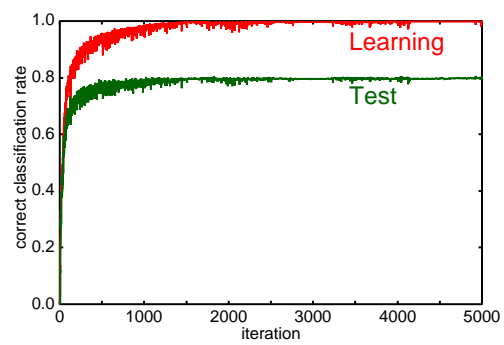

Fig. 8. Learning curves for segmentation.

Furthermore, the probability of correct and error classifications and their rate, that is $P_{c}, P_{e}$ and Rate, are shown in Tabel II. The probabilies were averaged over the iterations from 4,001 to 5,000. From these results, accuracy of the segmentation is almost the same as that of using all data. 
TABLE II

PROBABILITY OF CORRECT AND ERROR CLASSIFICATIONS AND THIR RATES FOR SEGMENTATION.

\begin{tabular}{|c||c|c|c||c|c|c|}
\hline \multicolumn{1}{|c||}{} & \multicolumn{3}{c||}{ TRaining data } & \multicolumn{3}{c|}{ Testing data } \\
\hline & $P_{c}$ & $P_{e}$ & Rate & $P_{c}$ & $P_{e}$ & Rate \\
\hline Segmentation & 99.7 & 0.1 & 0.99 & 79.7 & 10.5 & 0.88 \\
\hline No Segmentation & 100.0 & 0.0 & 1.00 & 78.0 & 0.0 & 1.00 \\
\hline
\end{tabular}

\section{Effects of Nonlinear Normalization}

Effects of the nonlinear normalization given by Eq.(1) on the mental task classification accuracy is investigated. For reference, linear normalization, by which the sample values are linearly normalized from 0 to 1 , is also used for reference. The segmentation is used, and 125 samples are reduced to 20 samples by averaging.

The learning curves are shown in Fig.9

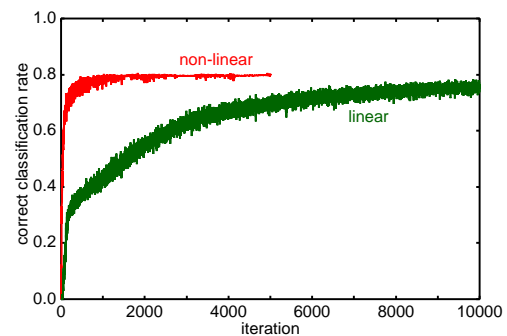

Fig. 9. Learning curves for nonlinear normalization.

Furthermore, the probability of correct and error classifications and their rate are shown in Table III. From these results, the nonlinear normalization can make convergence of the learning fast, and the probability can be also improved.

TABLE III

PROBABILITY OF CORRECT AND ERROR CLASSIFICATIONS AND THIR RATES FOR NONLINEAR NORMALIZATION.

\begin{tabular}{|c||c|c|c||c|c|c|}
\hline \multicolumn{1}{|c||}{} & \multicolumn{3}{c||}{ TRaining data } & \multicolumn{3}{c|}{ Testing data } \\
\hline Normalization & $P_{c}$ & $P_{e}$ & Rate & $P_{c}$ & $P_{e}$ & Rate \\
\hline Nonlinear & 99.7 & 0.1 & 0.99 & 79.7 & 10.5 & 0.88 \\
\hline Linear & 81.8 & 1.9 & 0.98 & 68.4 & 9.8 & 0.88 \\
\hline
\end{tabular}

\section{Threshold for Rejection}

When the winner output has small value, the estimation by the neural network is not accurate. Therefore, the result is rejected. In this section, effects of the threshold for rejection is investigated. The probability of correct and error classifications and their rates are listed in Table IV. By setting the threshold to be low, $P_{c}$ can be improved, however, $P_{e}$ is also increased at the same time. In some applications, $P_{e}$ should be minimized while the rejection can permitted to some extent. In these cases, high threshold is prefered. Like this, the optimum threshold is highly dependent on applications, to which the BCI system is applied.

\section{E. Comparison to Conventional Methods}

The same brain waves were used in [3]. The coefficients of a 6-th order auto-regression model are used for the neural
TABLE IV

Probability of CORRECT AND ERROR Classifications AND THIR RATES BY CHANGING THRESHOLD.

\begin{tabular}{|c||c|c|c|}
\hline Threshold & $P_{c}$ & $P_{e}$ & Rate \\
\hline 0.8 & 80.3 & 10.0 & 0.89 \\
\hline 0.6 & 81.7 & 11.9 & 0.87 \\
\hline 0.4 & 82.9 & 13.2 & 0.86 \\
\hline 0.2 & 83.7 & 14.2 & 0.86 \\
\hline 0.0 & 84.5 & 15.5 & 0.84 \\
\hline
\end{tabular}

network input. The data length of $10 \mathrm{sec}$ is also divided into $0.5 \mathrm{sec}$ segments. The probability of correct classifications is from $30 \%$ to $60 \%$ for four subjects. Therefore, the proposed method, which employs the amplitude response of the FFT, the nonlinear normalization, the averaging and the rejection, can provide higher probability of correct mental classification.

\section{CONCLUSION}

A neural network has been applied to the BCI problem. In order to improve accuracy of mental task classification, several kinds of pre-processing to generate the input data of the neural network. Compared with the conventional methods, the probability of correct classification has been increased.

\section{REFERENCES}

[1] Colorado State University: http://www.cs.colostate.edu/eeg/

[2] C. Anderson and Z. Sijercic, "Classification of EEG Signals from Four Subjects During Five Mental Tasks, "EANN'96, ed. by Bulsari, A.B., Kallio, S., and Tsaptsinos, D., Systems Engineering Association, PL 34, FIN-20111 Turku 11, Finland, pp. 407-414, 1996.

[3] J. R. Millan, J. Mourino, F. Babiloni, F. Cincotti, M. Varsta, and J. Heikkonen,'Local neural classifier for EEG-based recognition of metal tasks,"IEEE-INNS-ENNS Int. Joint Conf. Neural Networks, July 2000.

[4] G. Pfurtscheller and C. Neuper, " Motor imagery and direct braincomputer communication, " Proc. IEEE, vol. 89, no. 7, pp. 1123-1134, July 2001.

[5] K. R. Muller, C. W. Anderson, and G. E. Birch,” Linear and non-linear methods for brain-computer interfaces,'IEEE Trans. Neural Sys. Rehab. Eng., vol. 11, no. 2, pp. 165-169, 2003.

[6] B. Obermaier, G. R. Muller, and G. Pfurtscheller, "Virtual keyboard controlled by spontaneous EEG activity, "IEEE Trans. Neural Sys. Rehab. Eng., vol. 11, no. 4, pp.422-426, Dec. 2003.

[7] J. R. Millan, " On the need for on-line learning in brain-computer interfaces, " Proc. IJCNN, pp. 2877-2882, 2004.

[8] G. E. Fabiani, D. J. McFarland, J. R. Wolpaw, and G. Pfurtscheller, "Conversion of EEG activity into cursor movement by a brain-computer interface (BCI), "IEEE Trans. Neural Sys. Rehab. Eng., vol. 12, no. 3, pp. 331-338, Sept. 2004.

[9] G. Pfurtscheller, C. Neuper, C. Guger, W. Harkam, H. Ramoser, A. Schlögl, B. Obermaier, and M. Pregenzer, "Current trends in Graz braincomputer interface (BCI) research," IEEE Trans. Rehab. Engng., vol.8, pp.216-219, 2000.

[10] B. Obermaier, C. Neuper, C. Guger, and G. Pfurtscheller, "Information transfer rate in a five-classes brain-computer interface," IEEE Trans.Neural Sys. Rehab. Eng., vol.9, no.3, pp.283-288, 2001.

[11] C.W. Anderson, S.V. Devulapalli, and E.A. Stolz, "Determining mental state from EEG signals using neural networks," Scientific Programming, Special Issue on Applications Analysis, Vol.4, No.3, pp.171-183, Fall, 1995.

[12] K.Inagaki and K.Nakayama, "Mental task classification based on brain waves by using neural network", IEICE, Technical Report, Vol.105, No.174, SIP2005-54, pp.25-30, July 2005. (in Japanese)

[13] K.Inagaki and K.Nakayama,"On brain computer interface by using multilayer neural network", IEICE, 20th Signal Processing Symposium, Dec. 2005 (in Japanese). 